\title{
Attachment of an Electron-Withdrawing Fluorophore to a Cryptand for
}

Modulation of Fluorescence Signaling

Bamaprasad Bag and Parimal K. Bharadwaj*

Department of Chemistry, Indian Institute of Technology Kanpur, 208016, India

Email:pkb@iitk.ac.in

\section{Supplementary Information}

\section{Captions for the figures and tables:}

Fig. S1 FAB Mass spectrum of $\mathbf{L}$

Fig. S2 $\quad{ }^{13} \mathrm{C}$ NMR spectra of $\mathbf{L}$

Fig. S3 ${ }^{1}$ H NMR spectra of $\mathbf{L}$

Fig. S4 Absorption spectra of $\mathbf{L}$ in solvents varying polarity.

Table ST1 Absorption and $\varepsilon\left(\mathrm{dm}^{3} \mathrm{~mol}^{-1} \mathrm{~cm}^{-1}\right)$ of $\mathbf{L}$ in various solvents.

Fig. S5 Corrected fluorescence spectra of $\mathbf{L}$ in different solvents.

Table ST2 Absorption of $\mathbf{L}$ and in presence of various metal ions.

Fig. S6 Normalized photoluminescence spectra of $\mathbf{L}$ and it's $\mathbf{C d}(\mathrm{II})$ complex.

Table ST3 Fluorescence quantum yields in a titration of $\mathbf{L}$ with $\mathrm{Cd}(\mathrm{II})$.

Fig. S7 Corrected fluorescence spectra of $\mathbf{L}$ in presence of $\mathrm{Cd}(\mathrm{II})$ with variation of counter anions. 


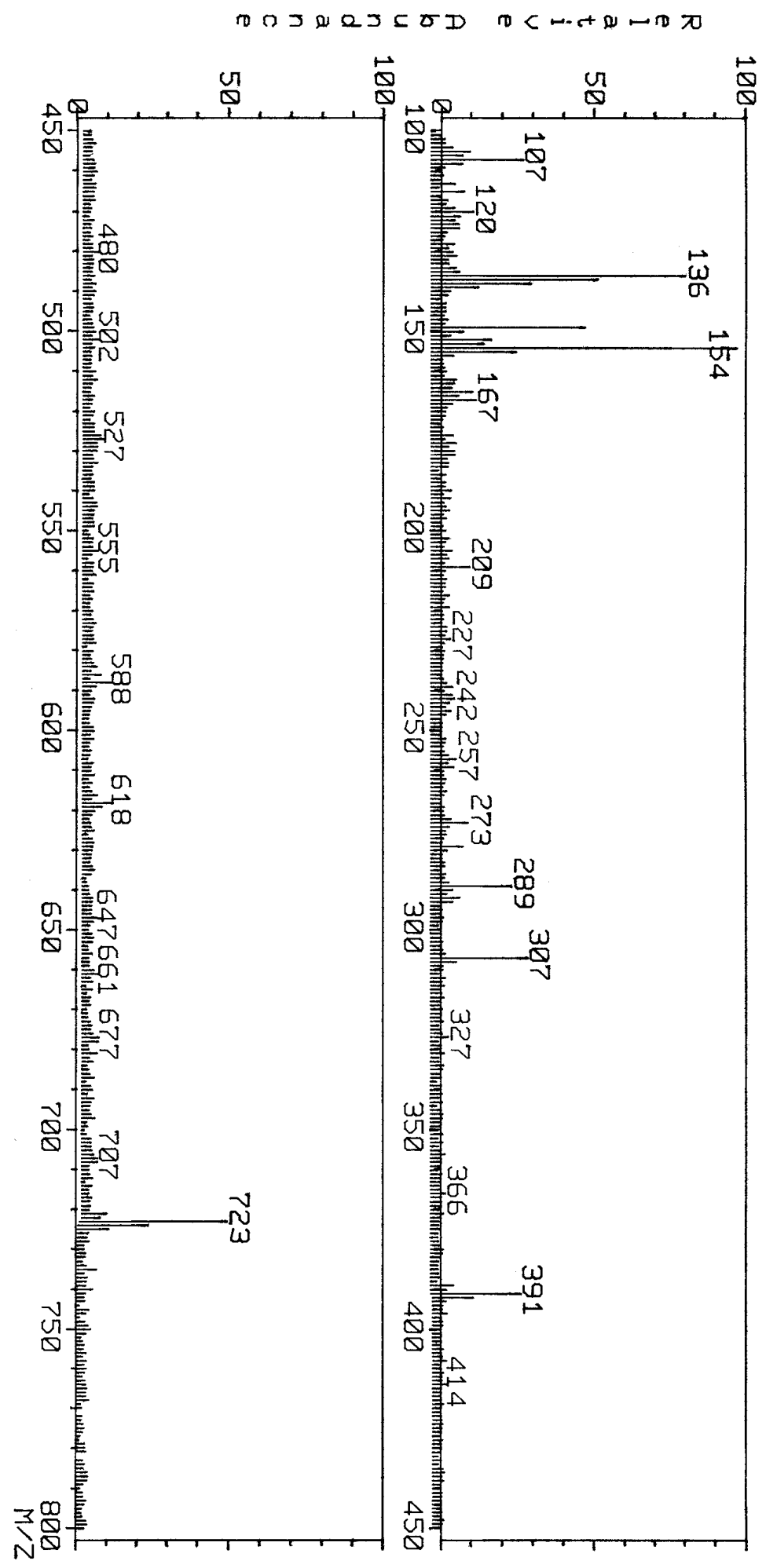

Fig. S1: FAB mass spectrum of $\mathbf{L}$ 


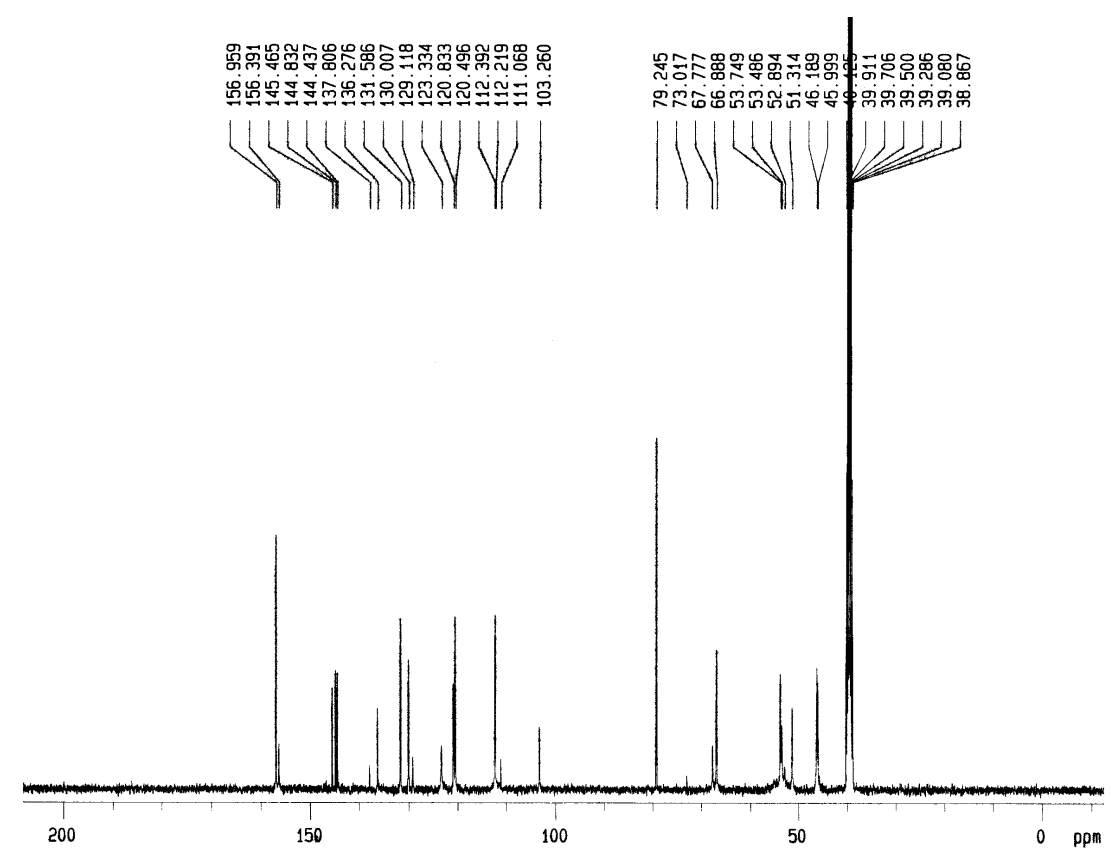

Fig. S2: ${ }^{13} \mathrm{C}$ NMR spectrum of $\mathbf{L}$
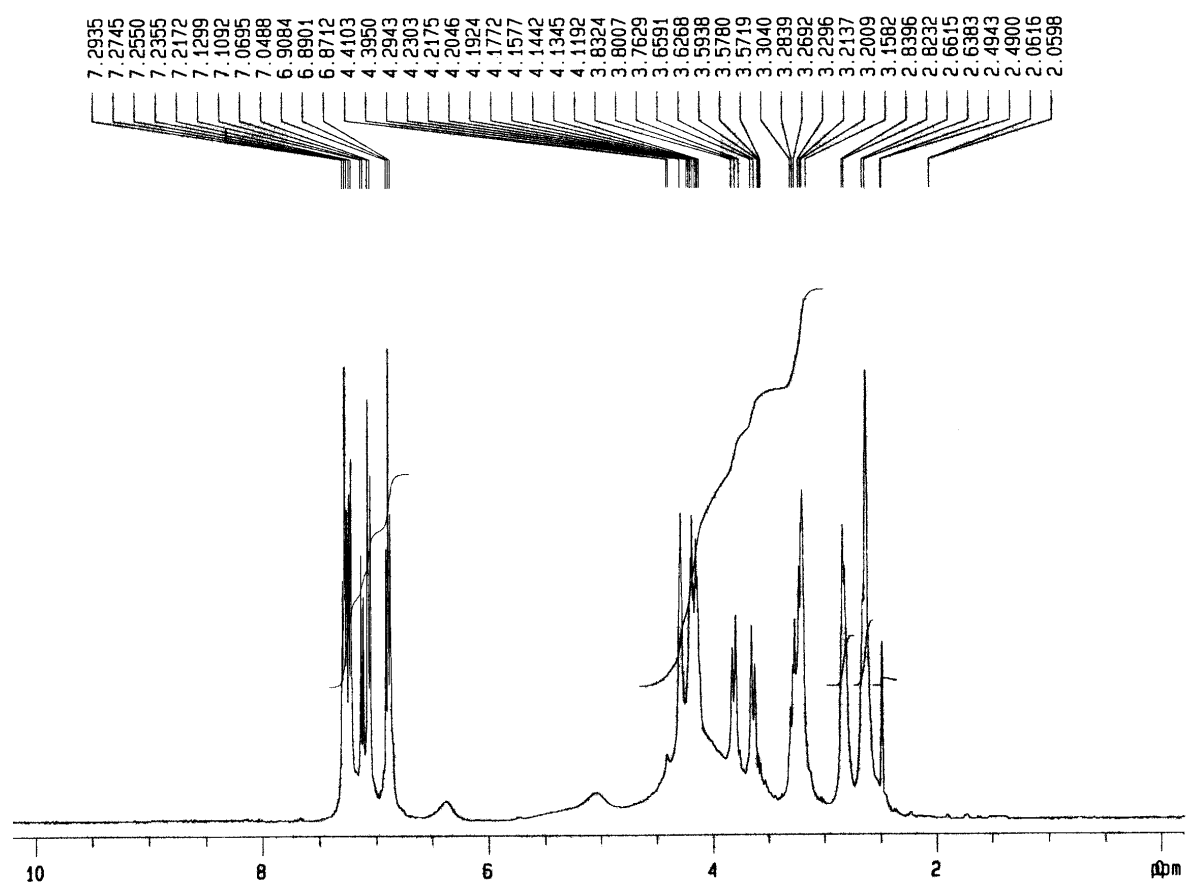

Fig. S3: ${ }^{1} \mathrm{H}$ NMR spectrum of $\mathbf{L}$ 


\section{Absorption spectra of $L$ in different solvents:}

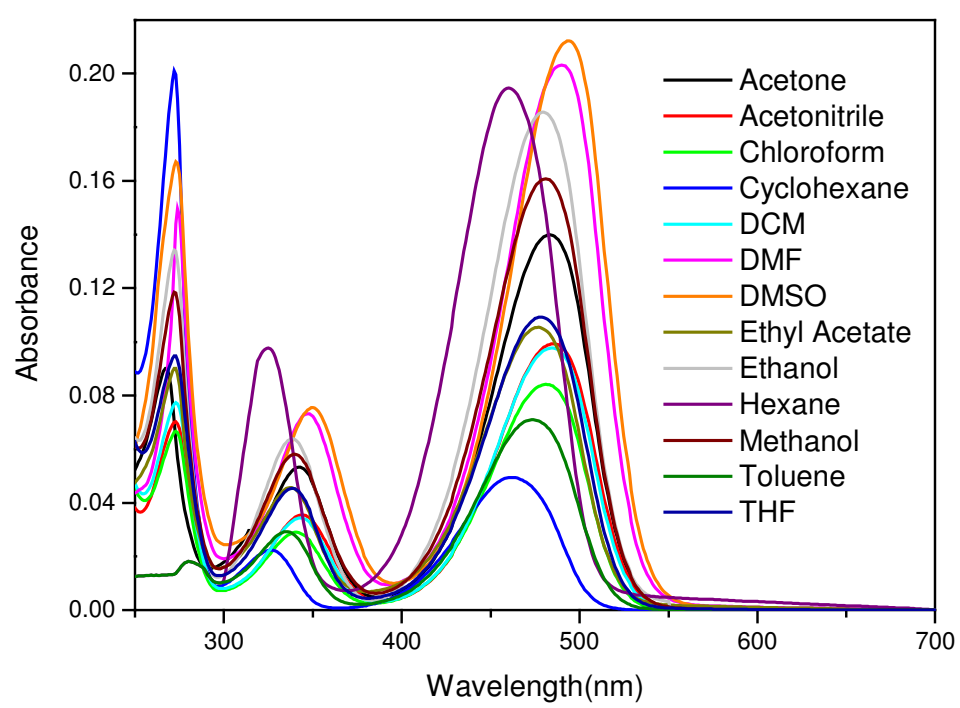

Fig. S4: Absorption spectra of $\mathbf{L}$ in various solvents at $\sim 1 \times 10^{-5} \mathrm{M}$ concentration.

Table ST1: Absorption and molar extinction coefficients of $\mathbf{L}$ in different solvents.

\begin{tabular}{lllc}
\hline Solvents & & $\lambda, \mathrm{nm}\left(\varepsilon, \mathrm{dm}^{3} \mathrm{~mol}^{-1} \mathrm{~cm}^{-1}\right)$ \\
\hline Hexane & $460(\mathrm{n} \mathrm{d})$ & $325(\mathrm{n} \mathrm{d})$ & $272(\mathrm{n} \mathrm{d})$ \\
Cyclohexane & $461(\mathrm{n} \mathrm{d})$ & $327(\mathrm{nd})$ & $272(\mathrm{n} \mathrm{d})$ \\
Toluene & $474(8909)$ & $335(3675)$ & $280(2280)$ \\
1,4 -dioxane & $475(6875)$ & $336(\mathrm{~s})$ & $278(17570)$ \\
Ethyl acetate & $476(12903)$ & $337(5208)$ & $273(10668)$ \\
Ethanol & $479(23234)$ & $338(8477)$ & $272(17556)$ \\
THF & $479(13034)$ & $338(5305)$ & $272(9916)$ \\
Methanol & $481(20185)$ & $340(7298)$ & $272(14888)$ \\
Chloroform & $481(10561)$ & $341(3640)$ & $273(8371)$ \\
Dichloromethane & $483(12253)$ & $343(4318)$ & $273(9711)$ \\
Acetone & $483(17558)$ & $342(6681)$ & - \\
Acetonitrile & $485(12464)$ & $344(4455)$ & $273(8816)$ \\
Dimethyl formamide & $490(25503)$ & $347(9198)$ & $274(18774)$ \\
Dimethyl sulfoxide & $494(26632)$ & $350(9482)$ & $273(20970)$ \\
\hline
\end{tabular}




\section{Emission spectra of $L$ in various solvents:}

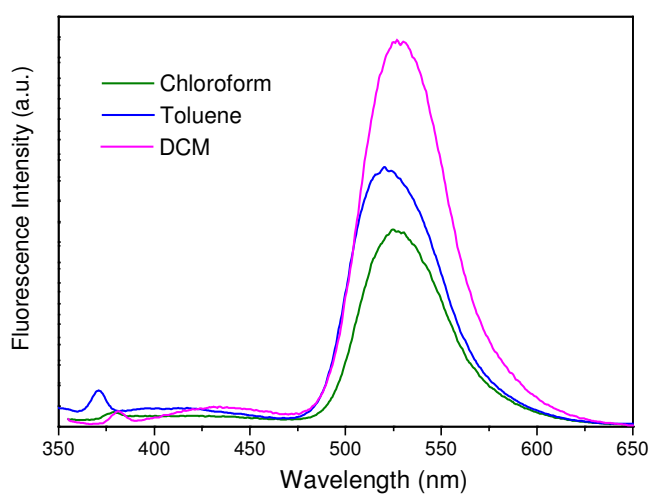

(a)

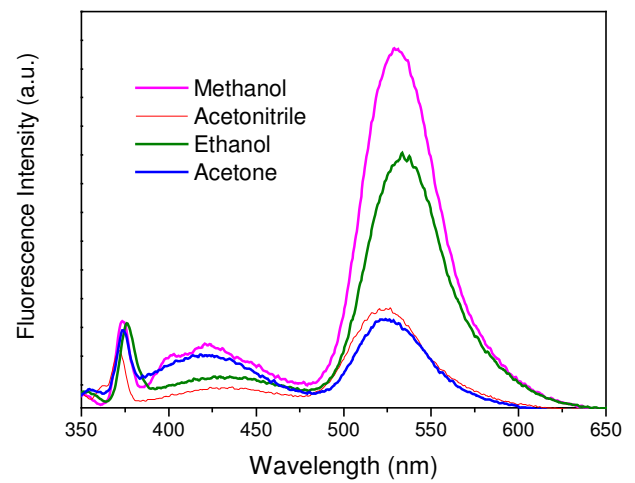

(b)

Fig. S5: Fluorescence spectra of $\mathbf{L}$ in (a) non-polar solvents (b) polar solvents. The RT spectra were taken at $\sim 1 \times 10^{-6} \mathrm{M}$ concentration.

Table ST2: Absorption of $\mathbf{L}$ and in presence of various metal ions in dry THF:

\begin{tabular}{clll}
\hline Input & $\lambda[\mathrm{nm}]\left(\varepsilon\left[\mathrm{dm}^{3} \mathrm{~mol}^{-1} \mathrm{~cm}^{-1}\right]\right)$ \\
\hline- & $479(13034)$ & $338(5305)$ & $272(9916)$ \\
$\mathrm{Mn}(\mathrm{II})$ & $473(11703)$ & $332(5804)$ & $272(17947)$ \\
$\mathrm{Fe}(\mathrm{II})$ & $472(10979)$ & $330(5153)$ & $261(10233)$ \\
$\mathrm{Co}(\mathrm{II})$ & $475(12536)$ & $335(5439)$ & $272(11885)$ \\
$\mathrm{Ni}(\mathrm{II})$ & $472(12020)$ & $334(5262)$ & $271(12071)$ \\
$\mathrm{Cu}(\mathrm{II})$ & $470(10444)$ & $332(6831)$ & $267(9870)$ \\
$\mathrm{Zn}(\mathrm{II})$ & $472(11512)$ & $334(5822)$ & $272(11196)$ \\
$\mathrm{Ag}(\mathrm{II})$ & $475(12340)$ & $336(5458)$ & $274(10419)$ \\
$\mathrm{Pb}(\mathrm{II})$ & $474(11319)$ & $335(5056)$ & $275(14114)$ \\
$\mathrm{Cd}(\mathrm{II})$ & $470(12127)$ & $332(5284)$ & $266(5562)$ \\
$\mathrm{H}^{+}$ & $472(10170)$ & $336(5140)$ & $272(12110)$ \\
2,6-di tert.-butyl pyridine & $478(13218)$ & $336(4980)$ & $272(12090)$ \\
\hline
\end{tabular}


Photoluminescence spectra (normalized) of L and It's Cd(II) complex:

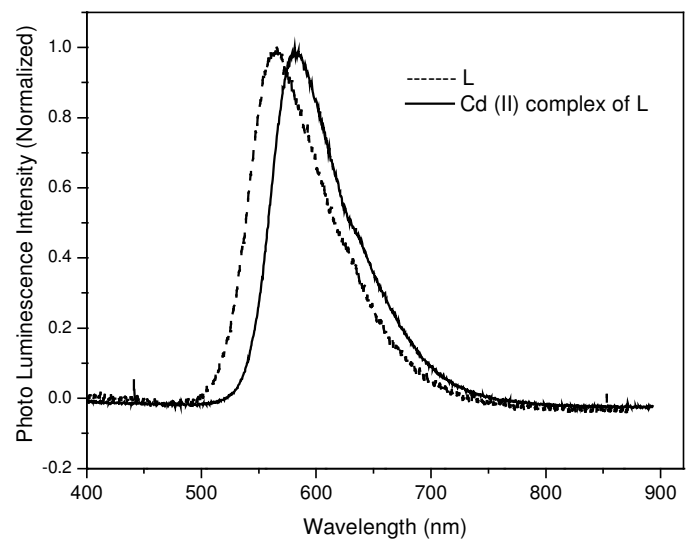

Fig. S6: Normalized photoluminescence spectrum of the ligand L and it's Cd(II) complex in solid state. The radiative charge transfer decay of the excited state of the ligand occurs at $566 \mathrm{~nm}$ which is $\sim 14 \mathrm{~nm}$ red shifted in it's Cd(II) complex.

Table ST3: Fluorescence output of $\mathbf{L}$ as a function of titration with $\mathrm{Cd}(\mathrm{II})$.

\begin{tabular}{lll}
\hline $\begin{array}{l}\text { Equivalents of } \\
\text { Cd(II) added }\end{array}$ & $\begin{array}{l}\text { Fluorescence } \\
\text { quantum yield, } \phi_{\mathrm{F}}\end{array}$ & $\begin{array}{l}\text { Fluorescence } \\
\text { Enhancement } \\
\text { Factor }(\mathrm{FE})\end{array}$ \\
\hline nil & 0.00189 & 1 (standard) \\
0.1 & 0.01156 & 6 \\
0.2 & 0.02028 & 11 \\
0.3 & 0.03861 & 20 \\
0.4 & 0.04963 & 26 \\
0.5 & 0.06978 & 37 \\
0.6 & 0.11619 & 62 \\
0.7 & 0.2166 & 115 \\
0.8 & 0.3048 & 161 \\
0.9 & 0.3932 & 208 \\
1.0 & 0.4579 & 242 \\
1.1 & 0.4579 & 242 \\
1.3 & 0.4577 & 242 \\
1.5 & 0.4579 & 242 \\
1.8 & 0.4580 & 242 \\
2.0 & 0.4578 & 242 \\
\hline
\end{tabular}

Fluorescence enhancement (FE) upon titration of $\mathbf{L}$ with $\mathbf{C d}\left(\mathrm{ClO}_{4}\right)_{2} \cdot \mathbf{x H}_{2} \mathrm{O}$ in dry THF at ligand concentration $=3.2 \times 10^{-6} \mathrm{M}, \lambda_{\mathrm{ex}}=338 \mathrm{~nm}$, RT. The plot of $\phi_{\mathrm{F}} v s$. equivalents of $\mathrm{Cd}(\mathrm{II})$ added shows 1:1 metal inclusion. 


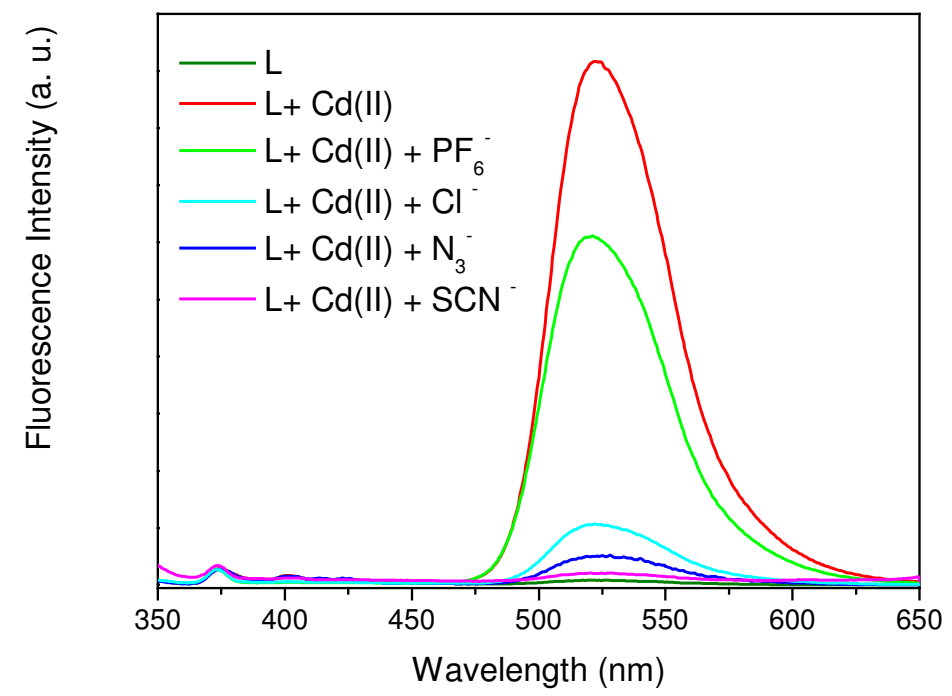

Fig. S7: Fluorescence spectra of $\mathbf{L}$ alone, in presence of $\mathrm{Cd}(\mathrm{II})$ and then by adding different counter anions in dry THF. $\lambda_{\mathrm{ex}}=338 \mathrm{~nm}$ at $3.2 \times 10^{-6} \mathrm{M}$ concentration. 